\title{
Inter-Machine Exchange of Real Time in Distributed Computer Systems
}

\author{
Vladimir Bogatyrev 1,2,3[0000-0003-0213-0223], Stanislav Bogatyrev 1[0000-0003-0836-8515], \\ and Anatoly Bogatyrev ${ }^{1}[0000-0001-5447-7275]$ \\ ${ }^{1}$ JSC NEO Saint Petersburg Competence Center, St. Petersburg, Russia \\ anatolyg@nspcc.ru \\ https://www.nspcc.ru/en \\ ${ }^{2}$ ITMO University, Saint Petersburg, Russia \\ vabogatyrev@corp.ifmo.ru \\ ${ }^{3}$ Saint-Petersburg State University of Aerospace Instrumentation, Russia
}

\begin{abstract}
The possibilities of increasing the likelihood of timely service and reducing the average waiting time for requests for inter-machine exchange in distributed real-time computer systems are investigated. The analyzed effect is achieved as a result of redundant multi-way transmissions of packets that are critical to delays, which provide for the replication of transmitted packets with the task for each replica of the path (route) of the sequential passage of network nodes. The condition for the timeliness of the reserved transmissions is that the accumulated waiting in the queues of the nodes making up the path, at least for one of the replicas, does not exceed the maximum permissible time. An analytical model is proposed for estimating the average delays of multi-path redundant transmissions, when determined by the average delivery time of the first of the replicas transmitted in different ways. For requests critical to service delays, the influence of the frequency of reservation (replication) of requests on the probability of their timely service and the average waiting time accumulated at the nodes of the path for the replica delivered first was analyzed.
\end{abstract}

Keywords: Reservation, Multipath Transfers, Real-time, Average Waiting Time

\section{Introduction}

Currently, great interest is shown in ensuring reliability and low latency in data exchange networks, these tasks are being addressed, including in the framework of the concept of Ultrareliable and Low-Latency Wireless Communication [1-4]. The need to ensure these requirements for distributed processing and data transfer systems,

Copyright $(C) 2020$ for this paper by its authors. Use permitted under Creative Commons License Attribution 4.0 International (CC BY 4.0).

* Publication supported by RFBR grant №19-07-00844 
including real-time images, is key. The problem of ensuring reliability and security is an acute problem for the currently developing cyberphysical systems [5,6].

To reduce the average transmission delays in the network allows transport coding $[7,8]$, in which the message is fragmented with the transfer of fragments along different routes. As a result of encoding fragments with distortion or non-delivery of their parts, the message can be restored, which allows to reduce the costs associated with organizing retransmissions of undelivered fragments (but requires complicating the processing process associated with decoding and restoration of the message).

To reduce the negative impact of failures on the continuity of the computing process, reconfiguration is aimed at combining it with the migration of virtual machines $[9,10]$ in cluster data processing systems $[11,12]$ and multi-path routing in networks $[13,14]$. Both of these technologies provide for the creation of reserve resources in order to quickly restore functioning during reconfiguration after failures. So with multi-path routing, the main and backup paths (routes) of data transmission are prescribed. After failures of the nodes of the main path, switching to a predefined backup path is carried out.

To reduce the average transmission delays in the network allows transport coding $[7,8]$, in which the message is fragmented with the transfer of fragments along different routes. As a result of encoding fragments with distortion or non-delivery of their parts, the message can be restored, which allows to reduce the costs associated with organizing retransmissions of undelivered fragments (but requires complicating the processing process associated with decoding and restoration of the message).

To reduce the negative impact of failures on the continuity of the computing process, reconfiguration is aimed at combining it with the migration of virtual machines $[9,10]$ in cluster data processing systems $[11,12]$ and multi-path routing in networks $[13,14]$. Both of these technologies provide for the creation of reserve resources in order to quickly restore functioning during reconfiguration after failures. So with multi-path routing, the main and backup paths (routes) of data transmission are prescribed. After failures of the nodes of the main path, switching to a predefined backup path is carried out.

A feature of the transmission of images in real time is the provision of delays in their delivery that do not exceed a certain maximum permissible value, as well as the fight against the spread of transmission delays (jitter) and their errors, which significantly reduce the reliability of communication and the quality of image perception, including video streams. To combat transmission errors, various methods are used, the main of which are noise-immune coding and retransmission schemes, however, the latter lead to an increase in delays, which leads to the use of protocols in real time without confirmation of delivery, increasing the timeliness of image transmission by reducing it reliability.

Real-Time Transport Protocol (RTP) and Resource Reservation Protocol (RSVP) are aimed at ensuring timely transmissions. RTP supports the timely delivery of data in real time to one or more subscribers. RSVP allows you to reserve network resources for the required quality of real-time transmissions over the RTP protocol.

Taking into account the specified specificity of image transmission, its quality is characterized by such indicators as average delivery delays, variance of delivery delays, 
delivery probability, taking into account bit errors of transmissions. For real-time transmissions, the key quality indicator is the probability of delivery within the maximum allowable time.

Reliability and timeliness of query execution in a redundant information and communication system (server cluster or switching nodes) can be improved as a result of redundant service of copies (replicas) of requests with the issuance of one completed copy (for example, the first issued in time) $[15,16]$.

Multipath backup service, provides for replication of requests) with the task for each replica of the path (route) of the sequential passage of nodes, systems [15].

The concept of ensuring the timeliness and reliability of the service under consideration is based on multi-path routing technologies $[13,14]$, multicast transmissions, and broadcast services [17], dynamic query distribution [18, 19] and clustering [20].

The condition for the success of redundant transmissions is that the accumulated total waiting in the queues of the nodes making up the path does not exceed the maximum permissible time for at least one of the copies. The quality indicator of real-time transmission systems is the probability of timely service of requests for inter-machine exchange and the average time of implementation of such an exchange [15].

The purpose of the article is to create an analytical model for estimating the average delays of multi-path redundant transmissions through a sequence of nodes that make up the paths when determining the required time for delivery of the first of the replicas transmitted over different paths.

The developed model is designed to assess the effectiveness and determine the feasibility of redundant multi-way transmissions depending on the frequency of their reservation, the intensity of the flow of requests and their criticality to delays in the queues of nodes making up the reserved paths. Similar problems must be solved in the modeloriented design of distributed computer systems [21-23], which function in real time, including distributed image processing and image streaming.

\section{Multipath Redundant Transmission Model}

Consider a system in which $\mathrm{k}$ replicas are created when generating requests for the transmission of a packet through the network. For each replica, the path of sequential passage of $m$ network nodes is registered. All paths include the same number of nodes. When assigning a node to be included in the $i$-th path, one of the $n_{\mathrm{i}}$ nodes is selected.

We represent the nodes of the infocommunication system as single-channel queuing systems with an infinite queue of type M/M/1 [24, 25]. To simplify the model, we assume that the $i$-th nodes of all paths are identical as queuing systems.

Taking into account the fact that when passing a certain replica of the next node included in the path, the margin of the acceptable waiting time decreases on average by the total average waiting time in the queues of the previous nodes, the probability of timely delivery when passing all $\mathrm{m}$ nodes of the path specified for the replica will be calculated as 


$$
P=\prod_{i=1}^{m}\left(1-\frac{\Lambda k v_{i}}{n_{i}} e^{\left(\frac{\Lambda k}{n_{i}}-\frac{1}{v_{i}}\right)\left(t_{0}-\sum_{j=1}^{i} w_{j}\right)}\right)
$$

where $v_{\mathrm{i}}$ is the average transmission time of the packet replica of the $i$-th node of the path, $t_{0}$-maximum allowed total waiting time for query replicas in the list of all nodes on the path, $\Lambda$ is the intensity of the input stream of requests for packet transmission, $k \Lambda$ is the intensity of the reserved input stream of requests for transmission of packet replicas, $\Lambda_{j}=\Lambda k / n_{j}$ is the intensity of the stream of requests arriving at the $\mathrm{j}$-th node, and $w_{\mathrm{j}}$ is the average timeout for request replicas in it.

$$
w_{j}=\frac{\Lambda_{j} v_{j}{ }^{2}}{1-\Lambda_{j} v_{j}}
$$

The probability of not exceeding the permissible travel time $t$ of all $\mathrm{m}$ nodes of the path, at least for one of the $k$ replicas, provided that the number of nodes included in all the paths and their characteristics are the same, we calculate as

$$
R=1-(1-P)^{k}
$$

The average delays of multi-path redundant transmissions through a sequence of communication nodes that make up the paths when determining the desired time for the first replica delivered from different paths in time

$$
W=\int_{0}^{\infty}\left\{\left[1-\prod_{i=1}^{m}\left(1-\frac{\Lambda k v_{i}}{n_{i}} e^{\left(\frac{\Lambda k}{n_{i}}-\frac{1}{v_{i}}\right)\left(t-\sum_{j=1}^{i} w_{j}\right)}\right)\right]^{k}\right] d t
$$

\section{Calculation of the Probability-Time Characteristics of Redundant Transmissions}

In the calculations, we will assume that the average request service time at each node is $v=0.1 \mathrm{~s}$, and the maximum allowable total waiting time in the queues of all nodes making up the path is $t_{0}=0.5 \mathrm{~s}$.

In Fig. 1 shows the dependence of the probability of timely reserved service on the intensity of the input request stream $\Lambda$, and Fig. 2 on the multiplicity of reservation requests. In Fig. 1, with the reservation factor $k=1,2,3$, curves 1-3 correspond to the path passing through $m=5$ nodes, and curves 4-6 through $m=10$ nodes. In Fig. 2, curves $1-5$ represent the intensity of the input request stream $\Lambda=500,600,700,900$, $10001 / \mathrm{s}$, respectively, at $m=10$. The calculations were performed for $\mathrm{n}_{\mathrm{i}}=n=5$ nodes.

The presented calculations confirm the existence of an optimal multiplicity of packet reservation critical to the total delay in the queues of the nodes transmitting them. It can be seen from the graphs that with a reduced load on the system, the recommended multiplicity of reservation of critical to expect packets, at which the maximum probability of timely delivery of at least one of the packet replicas is achieved, increases. 
In Fig. 3 shows the dependence of the average total waiting time in queues of nodes included in the path, counted until the first replica is delivered along one of the paths, on the intensity of the input request stream $\Lambda$. Fig. 4 shows the dependence of the indicated time on the multiplicity of reservation requests. In Fig. 3, when the request reservation ratio is $\mathrm{k}=1,2,3$, curves $1-3$ correspond to the path passing through $\mathrm{m}=5$ nodes, and curves 4-6 through $\mathrm{m}=10$ nodes. In Fig. 4, curves $1-5$ correspond to path lengths equal to $m=9,10,11,12,15$ nodes at an input request stream intensity $\Lambda=1$ $(1 / \mathrm{s})$. The calculations were performed for $n_{\mathrm{i}}=n=5$ nodes.

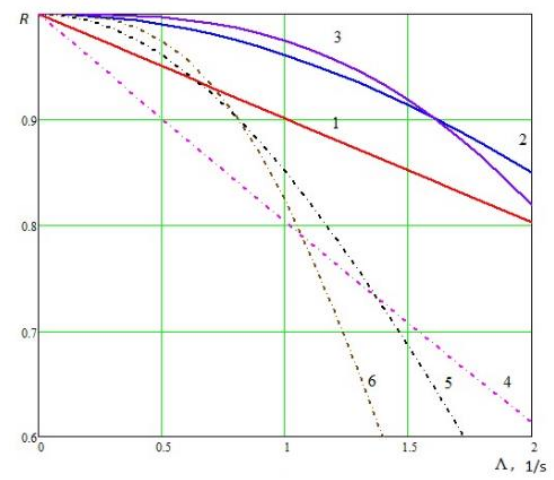

Fig. 1. Dependence of the probability of timely reserved service on the intensity of the input request stream

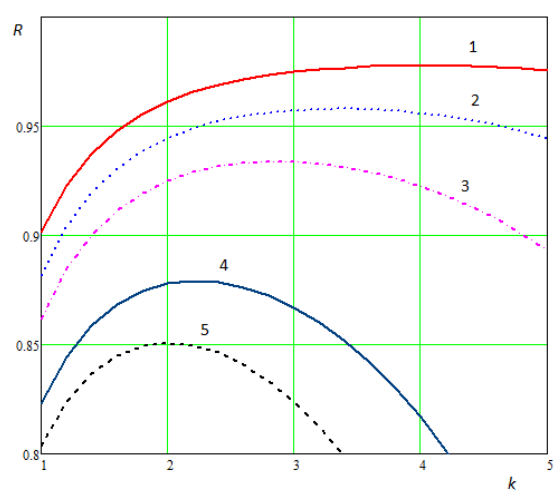

Fig. 2. The dependence of the probability of timely redundant service on the frequency of reservation requests 
6 V. Bogatyrev, S. Bogatyrev, A. Bogatyrev

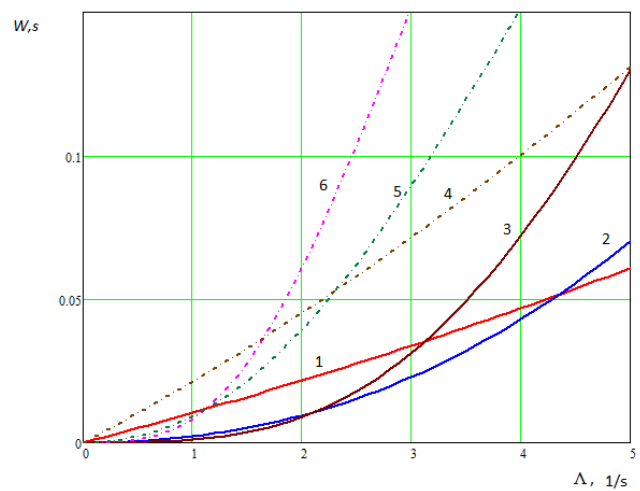

Fig. 3. Dependence of the average total waiting time on the intensity of the input request stream

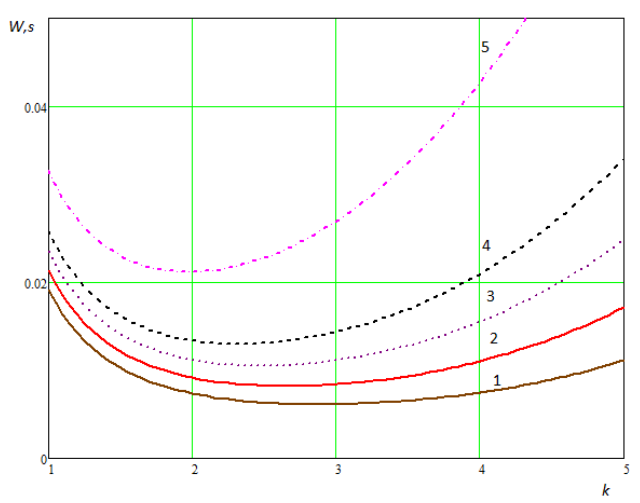

Fig. 4. Dependence of the average total waiting time on the frequency of reservation of requests

The established dependencies confirm the existence of an optimal multiplicity of reservation critical to the total delay in the request queues. The graphs show that with a decrease in the system load, the recommended redundancy rate at which the maximum probability of timely service and the minimum total average wait time in queues of nodes counted until the first replica is delivered along one of the paths is increased.

\section{Conclusion}

For multi-path redundant transmission systems, an analytical model is proposed for estimating the average total waiting time, counted until the first replica is delivered along one of the paths.

For requests critical to delays in queues, the influence of the frequency of reservation (replication) of requests on the probability of their timely service and on the average total delay in waiting for delivery of the first replica of time is analyzed.

The existence of an optimal multiplicity of reservation critical to the total delay in the request queues is shown. 


\section{References}

1. Siddiqi1, M., Yu, H., Joung, J. 5G Ultra-Reliable Low-Latency Communication Implementation Challenges and Operational Issues with IoT Devices Electronics 2019, 8, 981; doi:10.3390/electronics8090981 www.mdpi.com/journal/electronics

2. Ji, H., Park, S., Yeo, J., Kim, Y., Lee, J., Shim, B. Ultra-Reliable and Low-Latency Communications in 5G Downlink: Physical Layer Aspects. IEEE Wirel. Commun. 2018, 25, 124-130.

3. Sachs, J., Wikström, G., Dudda, T., Baldemair, R., Kittichokechai, K. 5G Radio Network Design for Ultra-Reliable Low-Latency Communication. IEEE Netw. 2018, 32, $24-31$.

4. Bennis, M., Debbah, M., Poor, H.V. Ultrareliable and Low-Latency Wireless Communication: Tail, Risk and Scale. Proc. IEEE 2018, 106, 1834-1853

5. Zakoldaev, D.A., Gurjanov, A.V., Shukalov, A.V., Zharinov, I.O. Functional safety of cyber-physical production of the Industry 4.0//IOP Conference Series: Materials Science and Engineering, 2020, Vol. 734, No. 1, pp. 012110

6. Gurjanov, A.V., Shukalov, A.V., Zakoldaev, D.A., Zharinov, I.O. Develop of reconfigurable manufacturing plant//Journal of Physics: Conference Series, 2020, Vol. 1515, No. 4, pp. 042060

7. Krouk, E., Semenov, S. Application of Coding at the Network Transport Level to Decrease the Message Delay // Proc. of 3rd Intern. Symp. on Communication Systems Networks and Digital Signal Processing. Staffordshire University, UK, 2002. pp. 109-112

8. Kabatiansky, G., Krouk, E., Semenov, S. Error Correcting Coding and Security for Data Networks. Analysis of the Superchannel Concept. Wiley, 2005. $288 \mathrm{p}$

9. Sahni, S., Varma, V. A hybrid approach to live migration of virtual machines Proc. IEEE Int. Conf. on Cloud Computing for Emerging Markets (CCEM 2012) Bengalore India pp 12-16 doi: 10.1109/CCEM.2012.6354587

10. Jin, H, Li, D., Wu, S., Shi, X,, Pan X. Live virtual machine migration with adaptive memory compression Proc. IEEE International Conference on Cluster Computing (CLUSTER '09). New Orleans, USA, 2009. Art. 5289170. doi: 10.1109/CLUSTR.2009.5289170

11. Machida, F., Kawato, M., Maeno, Y: Redundant virtual machine placement for fault-tolerant consolidated server clusters. In: IEEE Network Operations and Management Symposium, pp. 32-39. IEEE Press, Osaka (2010), doi: 10.1109/NOMS.2010.5488431.

12. 2020 71-85 Kim, S., Choi, Y. Constraint-aware VM placement in heterogeneous computing clusters. Cluster Comput 23, 71-85 (2020). https://doi.org/10.1007/s10586-019-02966-6

13. Merindol, P. Improving Load Balancing with Multipath Routing / P. Merindol, J. Pansiot, S. Cateloin // Proc. of the 17-th International Conference on Computer Communications and Networks, IEEE ICCCN 2008. - 2008. - P. 54-61.

14. Prasenjit, C., Tuhina, S., Indrajit, B. Fault-tolerant multipath routing scheme for energy efficient wireless sensor networksInternational Journal of Wireless \& Mobile Networks (IJWMN) Vol. 5, No.2,April 2013 pp 33-45

15. Bogatyrev, V.A., Bogatyrev, S.V., Bogatyrev, A.V. Model and Interaction Efficiency of Computer Nodes Based on Transfer Reservation at Multipath Routing // Wave Electronics and its Application in Information and Telecommunication Systems (WECONF 2019) 2019, pp. 8840647 . doi: 10.1109/WE-CONF.2019.8840647

16. Bogatyrev, V.A., Parshutina, S.A. Redundant Distribution of Requests Through the Network by Transferring Them Over Multiple Paths // Communications in Computer and Information Science - 2016, Vol. 601, pp. 199-207

17. Dudin, A.N., Sun, B.: A multiserver MAP/PH/N system with controlled broadcasting by unreliable servers. Automatic Control and Computer Sciences, No. 5, pp. 32-44 (2009). 
8 V. Bogatyrev, S. Bogatyrev, A. Bogatyrev

18. Bogatyrev, V.A. An interval signal method of dynamic interrupt handling with load balancing Automatic Control and Computer Sciences 2000, 34(6), pp. 51-57

19. Bogatyrev, V.A., Bogatyrev, S.V., Golubev, I.Yu. Optimization and the process of task distribution between computer system clusters / Automatic Control and Computer Sciences 2012, 46(3), pp. 103-111

20. Bogatyrev, V.A. Fault Tolerance of Clusters Configurations with Direct Connection of Storage Devices // Automatic Control and Computer Sciences - 2011, Vol. 45, No. 6, pp. 330337

21. Poymanova, E.D., Tatarnikova, T. M. Models and Methods for Studying Network Traffic. In 2018 Wave Electronics and its Application in Information and Telecommunication Systems (WECONF), pp. 1-5 (2018). doi:10.1109 / WECONF.2018.8604470

22. Kutuzov, O., Tatarnikova, T. On the acceleration of simulation modeling. Proceedings of 22nd International Conference on Soft Computing and Measurements, SCM 2019 (2019) p.45-47. DOI: 10.1109/SCM.2019.8903785

23. Astakhova, T., Verzun, N., Kolbanev, M., Shamin, A. A. Model for estimatingenergy consumption seen when nodes of ubiquitous sensor networks communicate information to each other. In Proceedings of the 10th Majorov International Conference on Software Engineering and Computer Systems, Saint Petersburg, Russia, December 20-21 (2018)

24. Kleinrock, L. Queueing Systems: Volume I - Theory. New York: Wiley Interscience. 1975 p. 417. ISBN 978-0471491101.

25. Kleinrock, L. Queueing Systems: Volume II - Computer Applications. New York: Wiley Interscience. 1976 p. 576. ISBN 978-0471491118. 